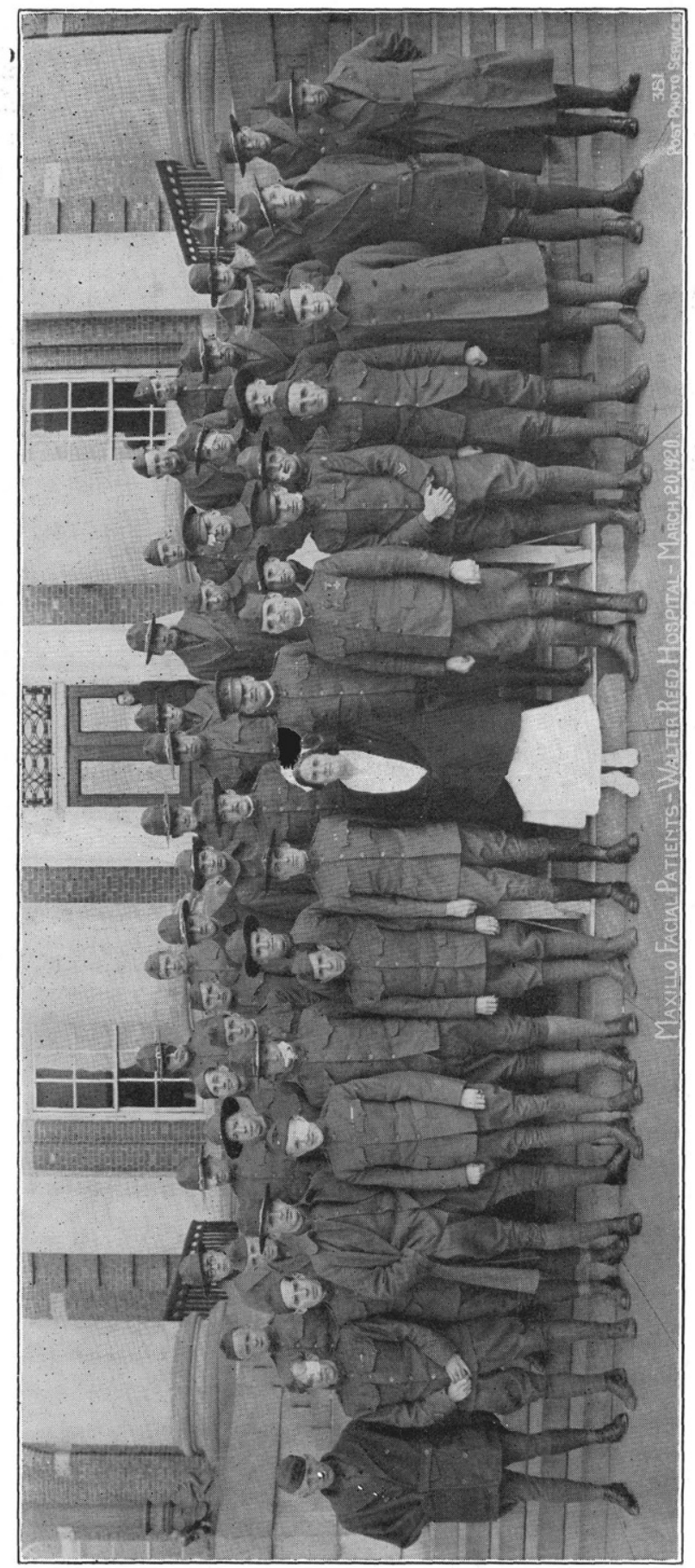




\title{
FREE SKIN GRAFTING IN THE MOUTH.
}

\author{
By Joseph D. Eby, Major, Dental Corp8, United States Army. \\ Walter Reed U. S. Army General Hospital, Washington, D. C.
}

(Published with permission of the Surgeon General, U. S. Army, who is not responsible for any opinion expressed or conclusions reached herein.)

$\mathrm{W}$

HEN a missile penetrates the maxillo-facial area and traverses the oral cavity, the mucous membrane lining of the lips and cheeks is lacerated, and the teeth intercepted are torn out with an extensive loss of alveolus and adjacent structures.

This condition leaves the areas of raw surfaces in contact in such a manner that the early treatment of the wound makes it very difficult to separate the surfaces and prevent adhesions owing to the fact that any material placed between the raw surfaces will usually seriously interfere with the treatment of other complications.

The result of healing causes the obliteration of that space between the buccal or labial gum ridges and the mucosa of the cheeks or lips which are commonly called the buccal or labial sulci.

These adhesions do not interfere usually with such work as the adjustment of splinting apparatus for the reduction of fractures, and in cases of loss of substance in the bone beneath, the additional protection afforded prevents the liability of entrance into the mouth during the bone grafting operation which lends a valuable point to the sometimes presence of these adhesions, so that they are not brought before the operator's attention as a serious defect to be corrected until the wound has thoroughly healed and seasoned and all other neces- sary repair has been completed up to the stage of inserting some form of prosthesis for the restoration of the loss of sub-structure to support the overlying tissues and for the restoration of lost teeth. There are probably a few exceptions to the above statement, one being that condition wherein these adhesions must be liberated primarily in order to gain free manipulation in the overlying tissues for plastic operations in the lips and cheeks. When the lips or cheeks are freed from the tissues beneath, the surfaces heal by granulation, and if any materials such as modeling composition, wax, paraffin, vaselined gauze, medicated gauze, vulcanite or metal are inserted between the raw surfaces, very painful sloughing and infection occurs which makes for an imperfect operation.

Much has been written in Plastic Surgery on the subject of free skin graftings, accredited usually to the early works of Drs. Thiersch and Ollier, but it was not until the demands were made as the result of Maxillo-Facial injuries in the European war that the possibilities of the success of free skin grafting in the mouth were made practical. One of the most valuable contributions to Oral Surgery evolved from the treatment of Maxillo-Facial injuries is the use of epithelium as an inlay to prevent the recurrence, shrinkage and to restore the full depth of the buccal and 
labial sulci which would otherwise be very stubborn conditions to overcome. This operation is commonly referred to as epithelial or skin inlays.

The advantages gained by this operation are:

1. Liberation of tissues to make plastic operations possible.

2. Restoration of function to the muscles of expression.

3. To improve facial appearance.

4. To replace prosthetically lost substructures.

5. For the insertion of dentures.

6. To close "perforations in the palate.

7. To liberate adhesions of the tongue.

8. To lengthen the lips.

9. To relieve Trismus.

There are no writings in Pre-War literature on this subject and such as have appeared to date in current periodicals are more or less in the abstract and do not deal definitely with the elements causing failure or methods which assure success.

This operation is applicable in all intra-oral adhesions whether they result from ulcerative lesions or from industrial, accidental or missile injurres.

The principles of success in this operation are:

1. Preoperative preparation.

2. Quick and accurate technic of operation.

3. Postoperative care and treatment. Preoperative Preparation.

There should be no infectious processes in or near the site of operation. In order to insure against all liabilities, the healed tissues should season for several months in order to eliminate all pathological changes and to produce highly vital tissues. The grafted skin must be lold securely in close contact against the raw surfaces covered, in order to cause a rapid agglutination and to prevent ingress of the fluids and micro-organisms in the mouth.

Some operators employ the method of adapting softened dental modeling compound into the crevas over the raw surfaces made by the incision, and after the skin graft is placed over this mould, it is secured to position by means of stay-sutures sewn across the exposed surface from one edge to the other. This plan is usually unsuccessful, and just here it may be well to explain the reasons. Mucous membrane granulates very rapidly and owing to the fact that nature's tendency in healing is to fill a deep cavity, this operation must be recognized as one opposed by, and attempting to overcome the natural laws of healing. When the mould or plumper is merely sutured in position the pressure of the overlying structures can easily press it slightly out of position, and when this occurs the graft is likewise pressed downward, causing either a loss of depth in the sulcus or failure to unite. Another reason why this method is contraindicated is the fact that it must be removed to make an impression for the permanent prosthesis, and it will be found very painful and tedious, to resuture the mould back in position to serve as a retainer until the denture is completed; but unless this is done, continued granulation will press downward such that when the denture is inserted it will depress the lips or distort the cheeks. For these reasons, this method should never be used. If the entire upper arch is edentulous the cranium should be employed by means of a head cap, or otherwise to effect the Kingsley principle of anchorage for the support of an intra-oral insert which can be tightened after the modeling composi- 
tion is adapted, and can also be removed, cleansed, replaced or tightened until the permanent denture is made to replace it.

If the lower arch is edentulous a chin cap attachment may be used similarly. When there are teeth remaining in the arches, mechanical devices attached to them are indicated. The basic indications for such an apparatus are security, ability to adjust and remove with provisions made for the secure attachment of modeling compound which may be softened and pressed into the deepest recesses after the adhesions are incised. To meet these demands the most ideal apparatus is Dr. V. H. Jackson's spring-clasp attachment of removable orthodontia apparatus. This appliance possesses such latitude and design, the spring clasps can be made very secure and loops of heavy wire can be formed imaginatively in the region to be epithelialised, so that the modeling compound may be inserted with a maximum of speed and accuracy, thus facilitating the operation at a very important stage. Another satisfactory method is to construct the partial denture with secure spring-clasp attachments then by perforating the border beneath the area to be operated, modeling compound can be added during the operation and later duplicated in vulcanite.

\section{Quick Accurate Technic of Opera- TION.}

If this operation is conducted in a clumsy or indifferent manner, failure is imminent. In preparation, the appliances to be used should be sterilized, or if of rubber, immersed in alcohol. The modeling compound of usual inamiacture cannot be immersed in cresol or alcohol, but can be readily treated in $1 / 1000$ bi-chloride of mercury. The two best materials for this use are
Kerr's Perfection Compound or the White Composition, manufactured by the Dental Mfg. Co., of London, Ltd. American agents, 220 West 42nd St., New York, N. Y. The latter material manipulates well when softened and its color value affords a much more accurate contrast in the mouth. The instruments necessary are a sharp curved scalpel and a very sharp razor or skingraft cutting instruments. Other materials required are sterile gauze $2 \times 2$ squares, or medium gauze sponges, sterile vaseline, hot water, boric acid solution, vaselined gauze dressings and $31 / 2 \%$ iodine. For a slight operation, local or conductive anesthesia is indicated. In very extensive conditions wherein a very great area of the face is undermined necessitating an equally large surface of skin graft, and where some delicate regional anatomy is involved, an intrapharyngeal general anesthetic is best. Whether this operation be performed in the dental chair or on an operating table, an uncompromising sterile technic must be followed. The patient's head should be covered with sterile cloth, the exposed surface around the mouth iodinized, and the mouth previously cleansed and rinsed with strong antiseptic, and preparations made to iodinize immediately before cutting into the tissues. In selecting the surface from whence to prepare the epithelium, the first indication is for a hairless surface situated where a scar will be less unsightly. For men, most satisfactory material can be obtained from the outer surface of the upper arm or from the thigh. With sterile coverings the skin should be bathed, washed in iodine and alcohol. It is to be preferred that the skin graft should be in one section in which there should be no perforations. The specimen of epithelium must be thin, and should be elevated thru the epidermis, 
a typical Thiersch graft. If the surface is covered with a thin film of stiff vaseline it is much easier to cut and control the portion removed.

With every possible preparation ready, the following steps should be executed with all speed, and the work of an assistant operator can reduce the time of operation at least $50 \%$.

Insert retractor in mouth, if possible, with scalpel incise deeply liberating the sulcus to a greater depth than normal, immediately thrust a hot boric sponge into the crevas, previously having the head posed so as to prevent the entrance of saliva. Soften the modeling compound previously arranged on a supporting apparatus preferably with dry heat in order to only soften the surface, press this quickly to position and with external pressure, mould it into the deepest recess of the incision (removing the hot gauze). Remove the apparatus with modeling compound thus fitted, and instantly replace a hot boric sponge. In the meantime the assistant operator should have procured the epithelium as follows: Grasp the arm very tightly in the left hand so as to make tension over the surface from which the epithelium is to be elevated or if a larger piece is required an assistant can flatten out the surface and make tension with two broad instruments. Lay the razor flat on the tight skin and make a backward and forward motion, loosening the epidermis of desired thickness and size. The vaseline on the outer surface of this epithelium again proves valuable by causing the specimen to adhere to the modeling compound, quickly withdrawing the gauze pack, the apparatus containing the epithelium can be thrust securely to position with the raw surface of the graft in contact with the raw surface of the incision. The graft should overlap all raw surfaces with some excess.

If saliva is pressed in between the two raw surfaces, the epithelium will fail to unite, it being expedient that the raw surfaces must be in intimate contact with no intermediate substance. The surface from whence the epithelium was procured should be dressed with gauze, saturated with vaseline, redressed daily until healed.

Postoperative Care and Treatment.

Apparatus holding the mould into position which supports the graft should not be removed until at least the fifth day, the mouth should be sprayed daily and receive the best prophylactic care. Upon the removal of the apparatus it will be found that the epithelium is aciierent to all raw surfaces and the excess can be removed along the borders. The surface should be sprayed, iodinized and the apparatus immediately replaced. The fact that this apparatus is replaceable becomes the cardinal factor at this stage for it serves as a positive retainer.

Impressions can be taken at any time for the construction of the permanent denture which must be of design which will fit into the depth of the sulcus, for it is only after several months of seasoning that obliterative granulation will be arrested, and the operation be made a permanent success.

This epithelium after having become re-vitalized at first retains its characteristic color and appearance, but after several months it gradually simulates the appearance of mucous membrane owing to the warm moist environment which it occupies. 


\section{Steps of Operation.}

Figure 1.

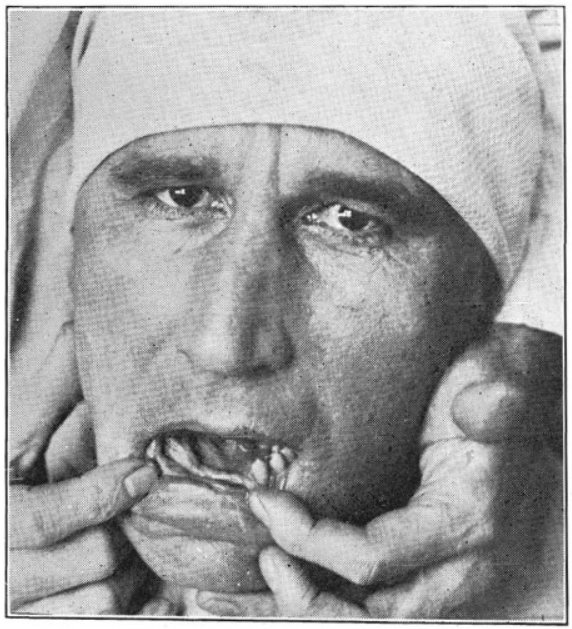

Illustrating the path of a machine gun bullet which passed thru the lower lip, eausing extensive loss of teeth, alveolus and lip, which upon healing obliterated the labial sulcus.

Figure 2 .

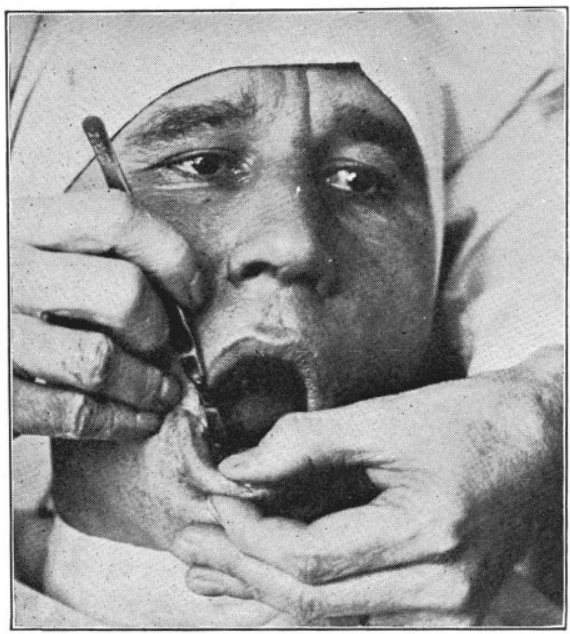

Incision to release lip from sub-structures.
Figure 3.

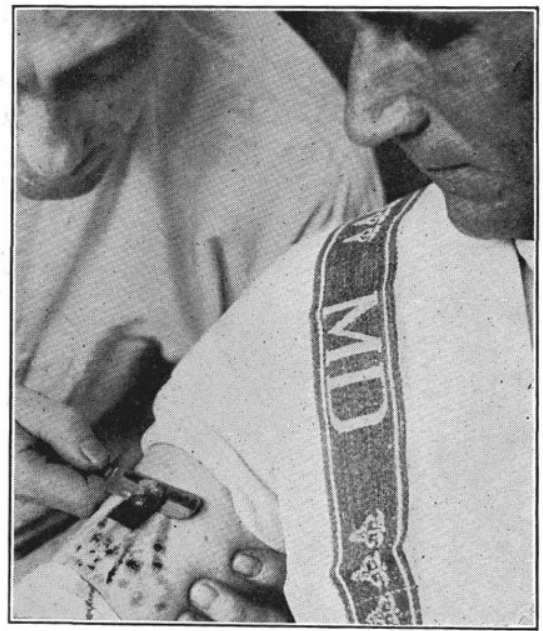

Shaving Thiersch graft from the arm.

Figure 4 .

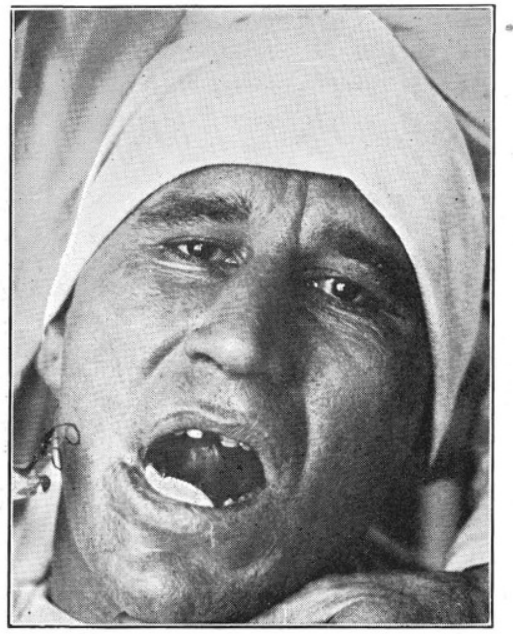

Illustrating control of saliva, the Jackson appliance with modeling compound, plumper moulded into the depth of the newly established sulcus with the raw surface of the Thiersch graft covering the composition and ready for insertion. 
Figure 5 .

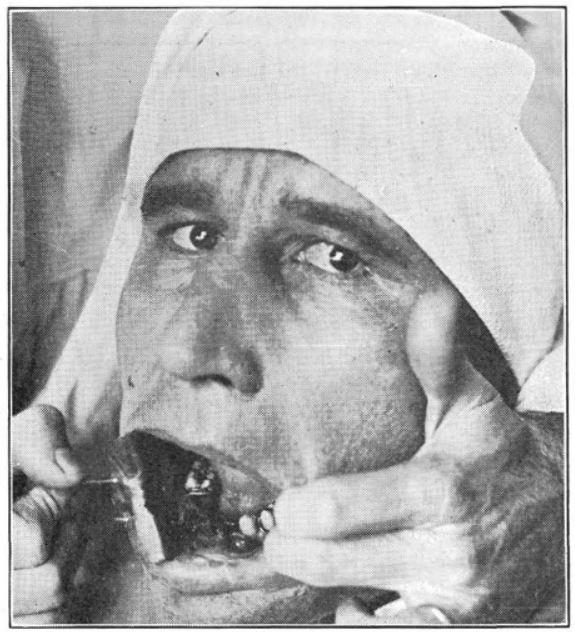

Apparatus in position with the graft held tightly against all raw surfaces.

Figure 6 .

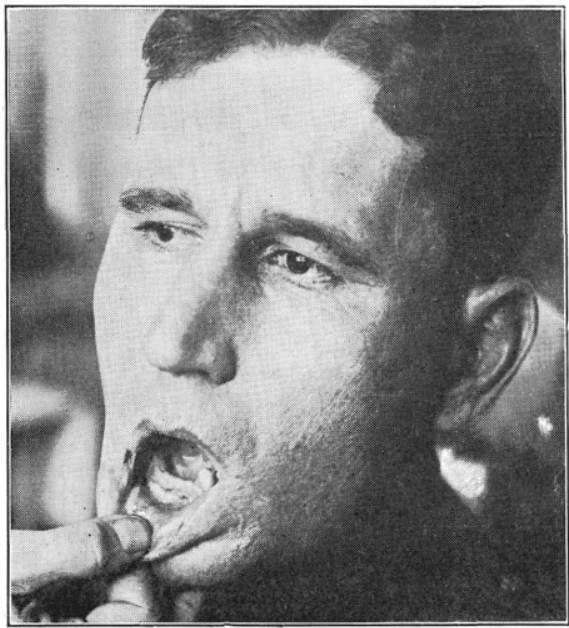

Partial view of graft after the removal of the apparatus five days after operation.
Figure 7 .

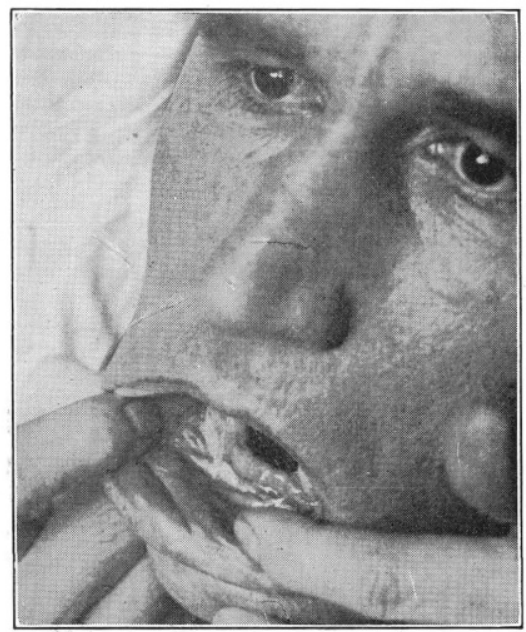

Close-up view of graft.

Figure 8 .

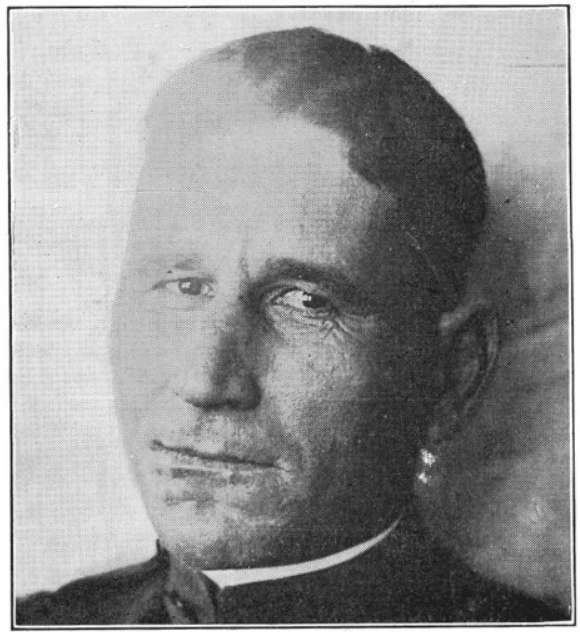

Completed case showing elevation of lip properly supported by a correct fitting denture beneath. 


\section{Epithelialization of Intra-Oral Adhesions, Edentulous, SUPERIOR MAXillae.}

Figure 9 .

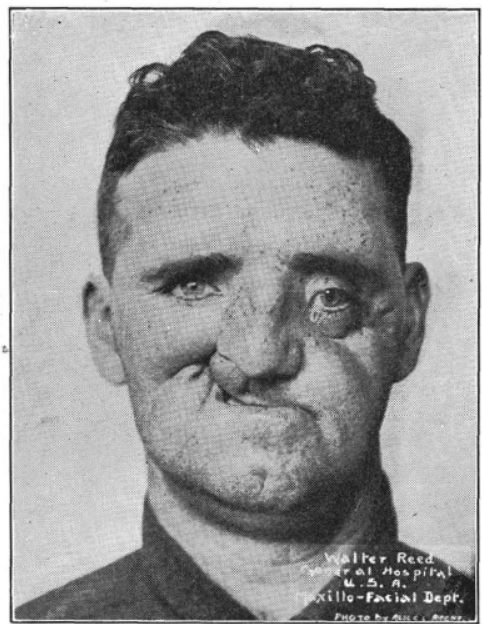

Figure 12.

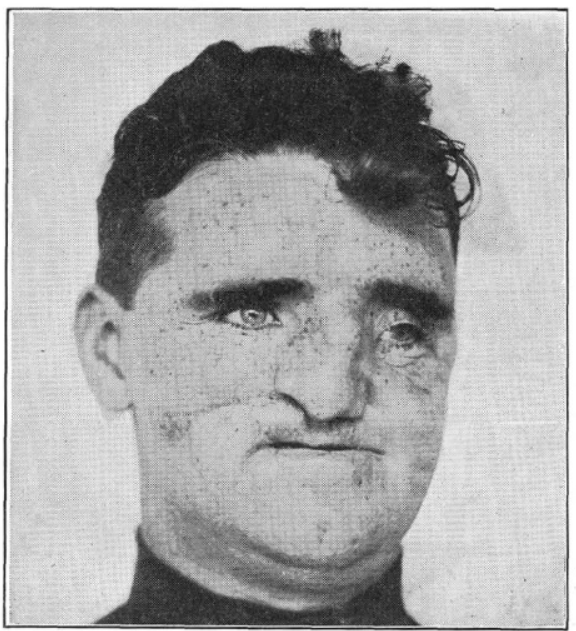

Figure 9. Entrance of high explosive fragment, left Malar ominence, exit thru right cheek destroying the facial, nasal and palatal processes of the superior maxillae. The overlying soft tissues collapsed by the contraction of thru and thru scar bands. All of these adhesions necessarily had to be loosened before the superficial plastic operation could be performed.

Figure 12. Result of operation.

Figures 10 and 11 .
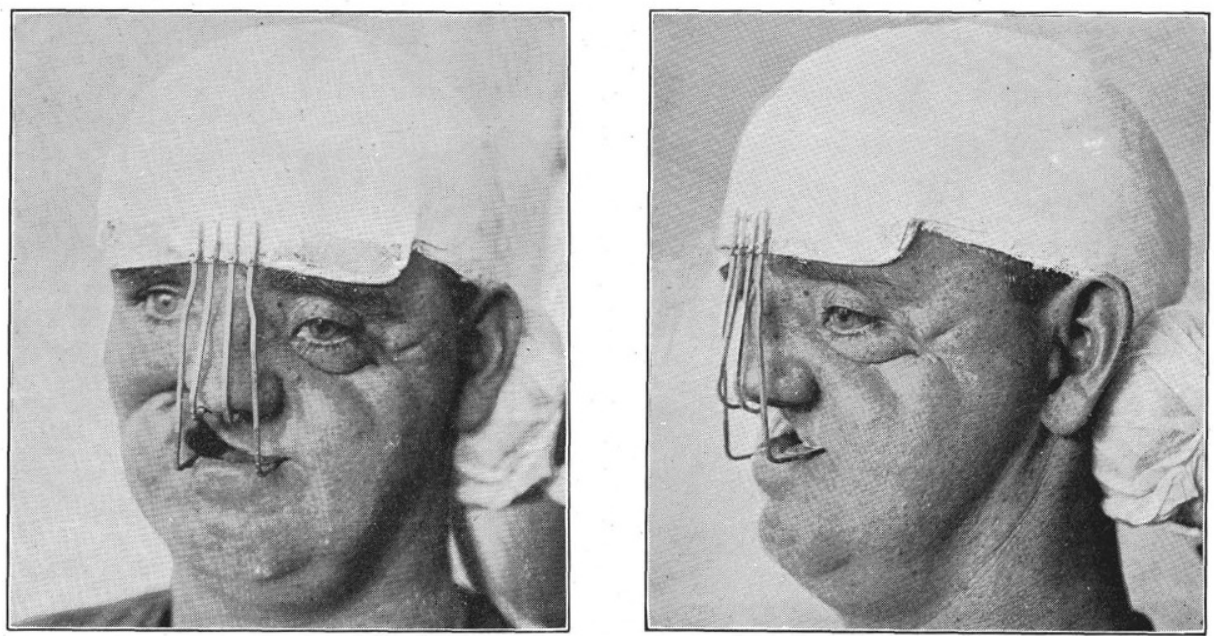

Cranial anchorage for intra-oral stage for the support of modeling composition. This apparatus consists of a metal plate swedged to fit the forehead with loops of (12 G.) wire soldered to the edges for reinforcement, and for the attachment of a plaster bandage cap To this plate was soldered four pieces of square tubing with set screws adjusted to secure the extensions in such a manner that they could be inserted, removed or tightened. Inasmuch as the entire middle-third of the face was to be undermined, it was considered necessary to provide special nasal supports. The intra-oral portion is similar to an edentulous upper impression tray with the exposed surface roughened to secure the compound. Operation general anesthetic, the line of the buccal and labial sulcus was first established by incision. The superior maxillary region was undermined, modeling compound was forced into position on the stage and epithelialized. The contracted scar right cheek was excised, edges sutured. 
Epithelialization, Intra-Oral Adhesions, Edentulous Mandible.

Figure 13.

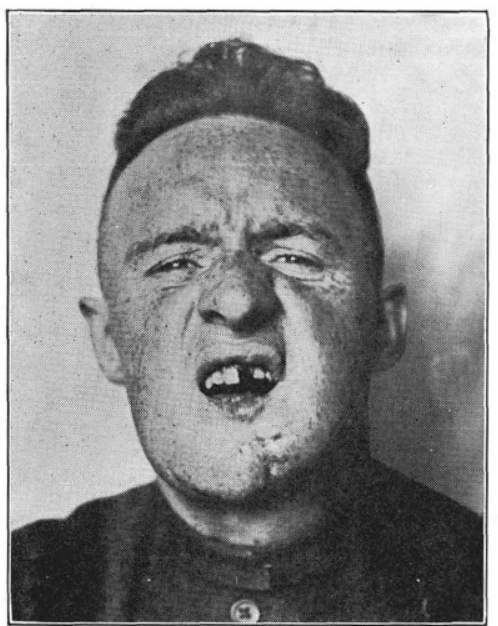

Completely adherent lower lip and cheeks.

Figure 15.

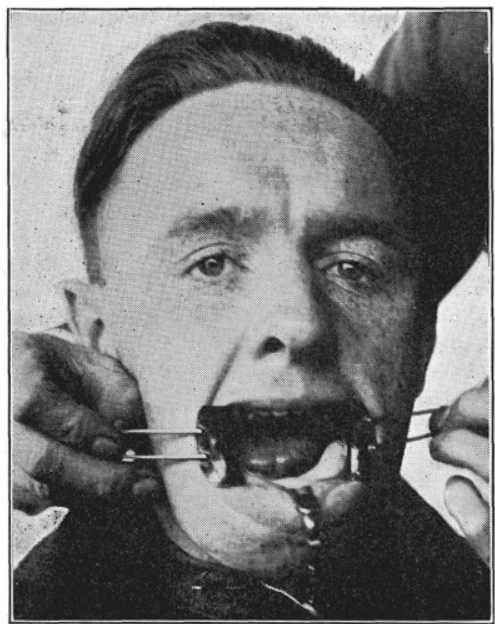

Operation performed local anesthetic, apparatus in position.
Figure 14.

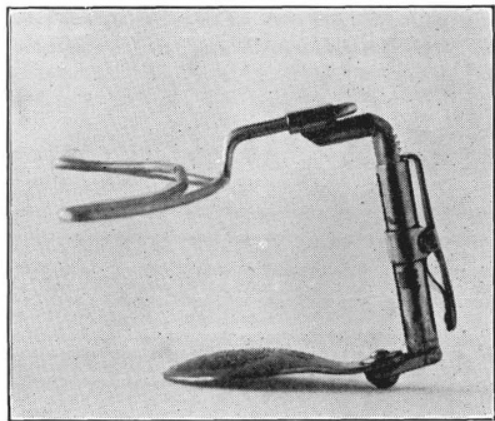

Pressure apparatus consisting of chin pad attached adjustable to an intra-oral loop of (12G.) wire with ratchet for insertion removable or tightening.

Figure 16.
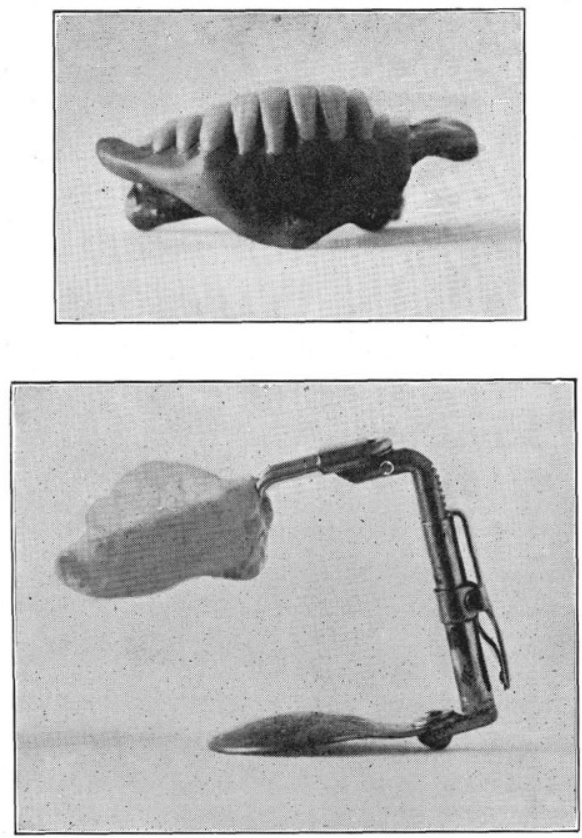

Apparatus with modeling compound mould which was worn until the denture illustrated was ready for insertion. 
Figure 17

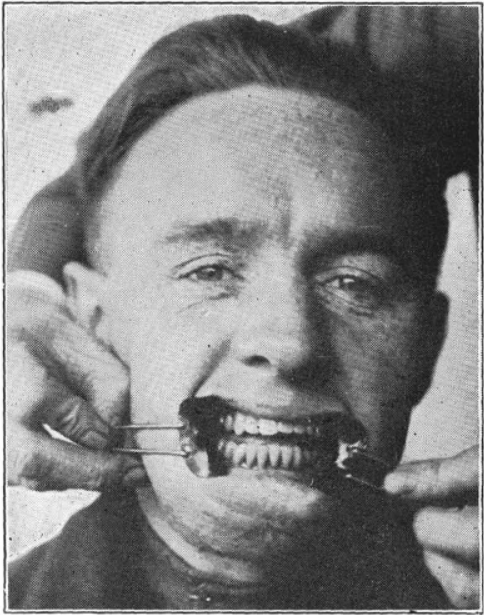

Denture in position,

\section{Epithelialization Prior to Plastic Operations.}

Figure 18.

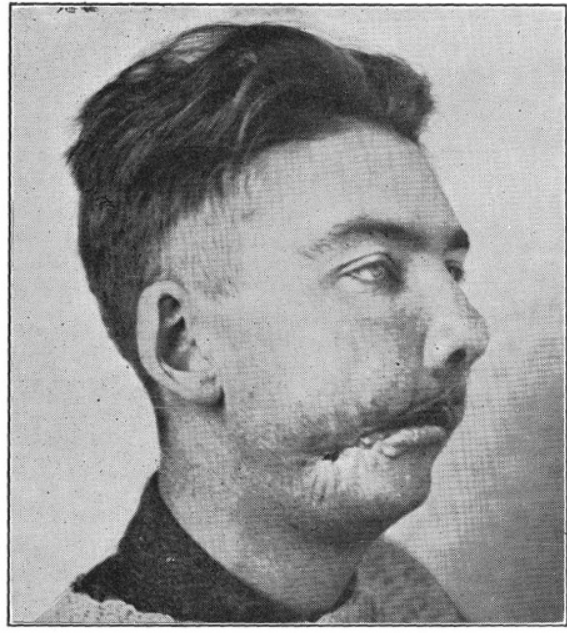

Path of high explosive fragment rendering botn upper and lower lips adherent to sub-structures region right corner of mouth.
Figure 19.

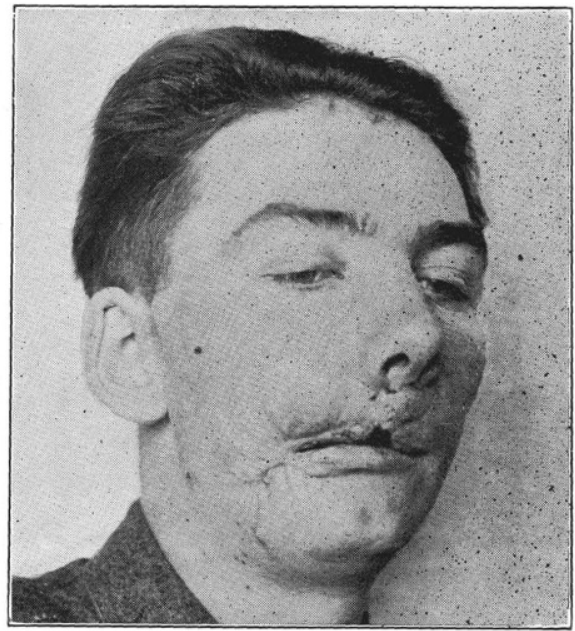

Lips liberated and prepared for plastic operation. 
Figure 20.
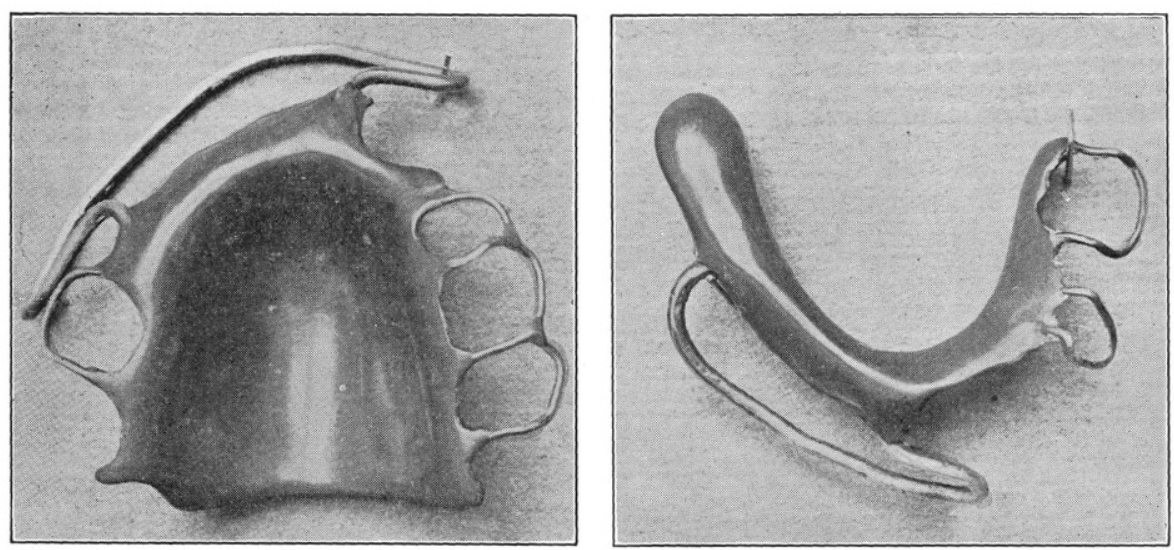

Modified Jackson apparatus to hold modeling compound moulds.

Jackson Apparatus for the Support of Skin Inlay.

Figures 21 and 22 .
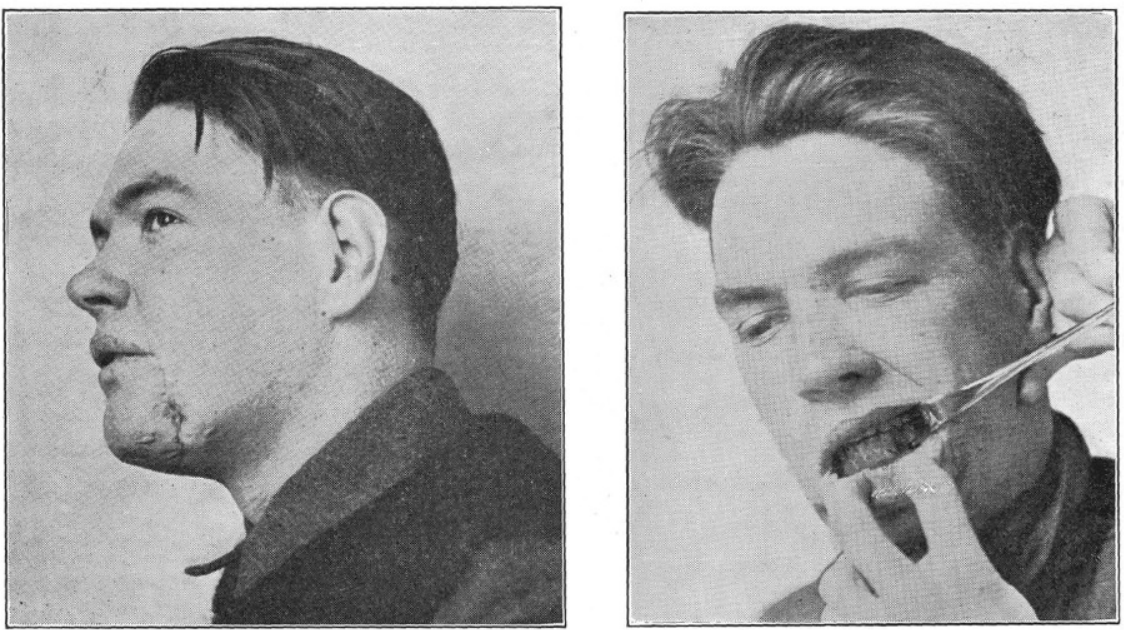

Sulcus entirely obliterated for space of three $\mathrm{Cm}$. left lower corner of mouth immobilizing the muscles of expression effecting a pseudo-paralysis. 
Figure 23.

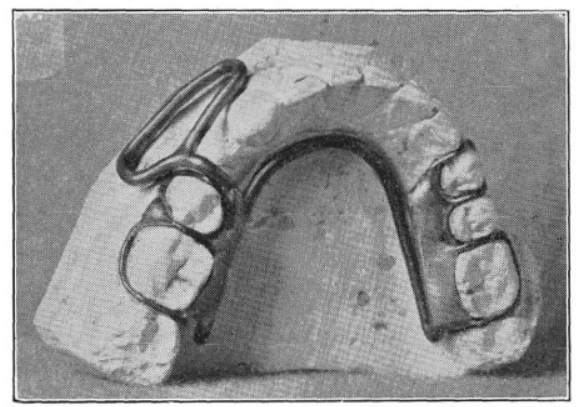

Jackson apparatus prepared to hold modeling compound plumper.
Figure 24.

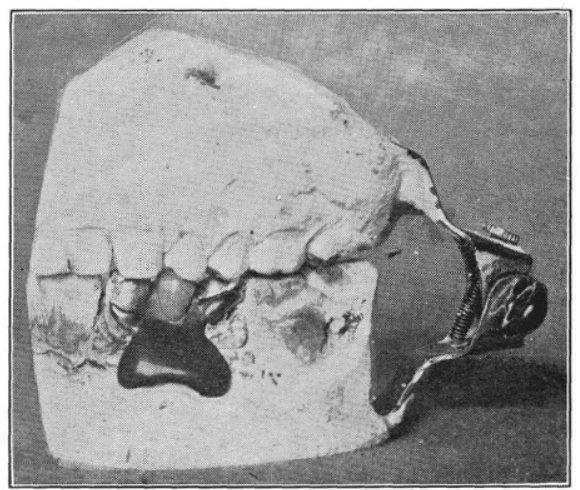

Denture prepared to retain the results accomplished.

Figure 25.

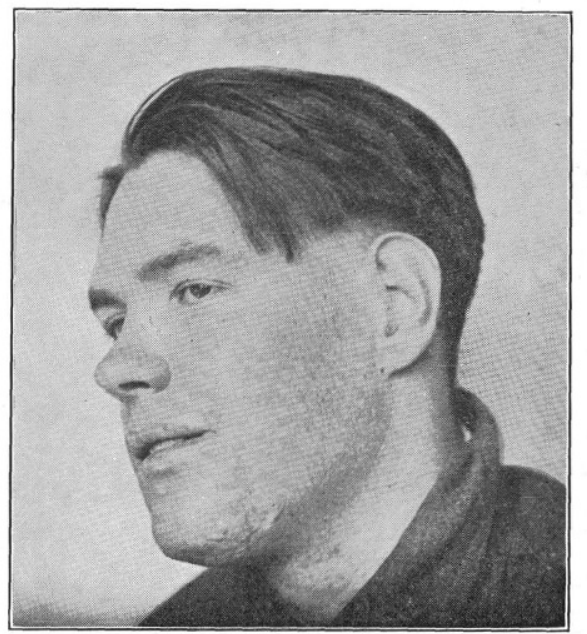

Illustrating the expression restored. 


\section{Modeling Compound Extensions on Dentures.}

Figure 28.

Figures 26 and 27.
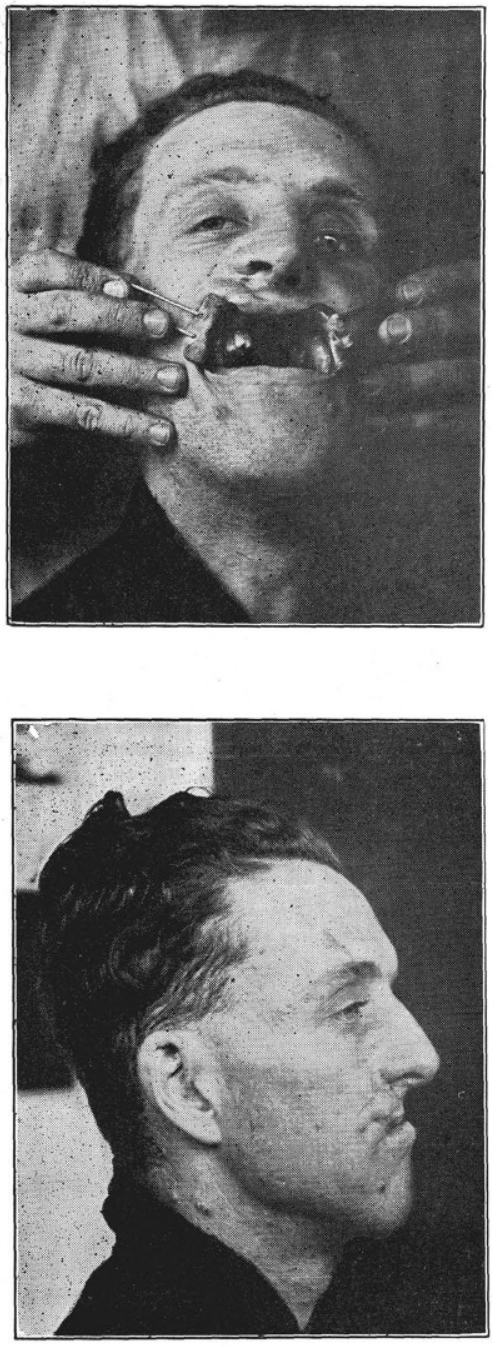

Path of missile, the extensive loss of alveolus upper labial sulcus obliterated, lip adherent.

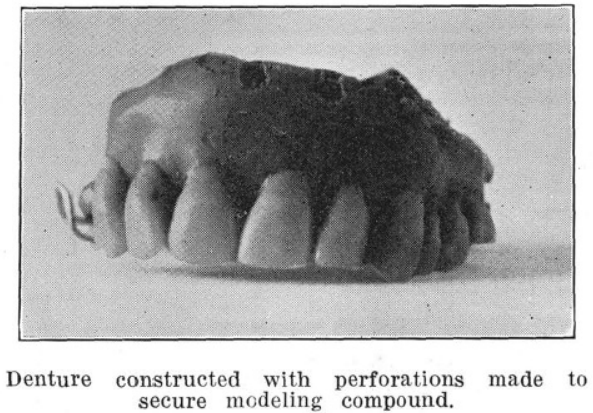

Figure 29.

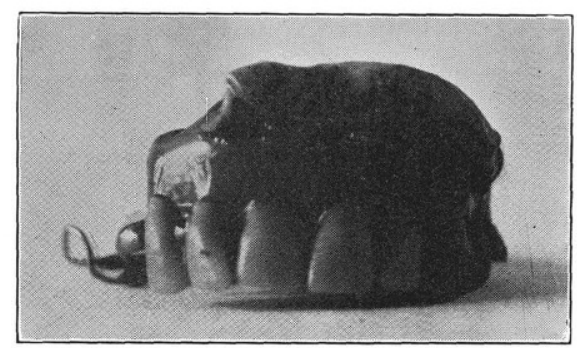

Modeling composition in position after operation which was later duplicated in vulcanite.

Figure 30 .

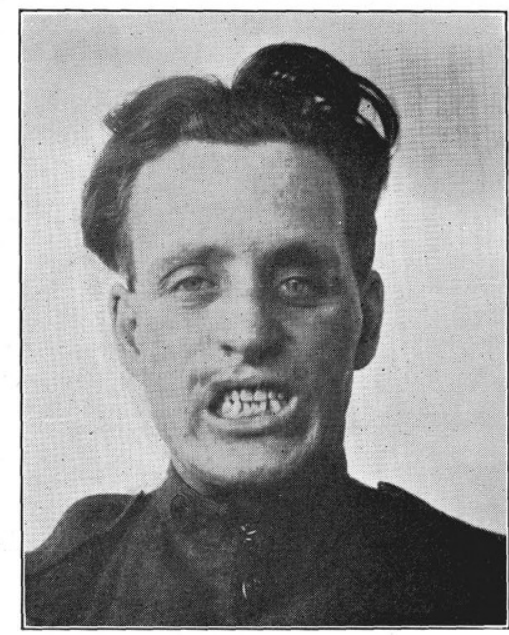

Denture in position, lips voluntarily retracted, the upper lip was lengthened sufficiently to close into normal lip-line contact with the lower. 
Figure 31.

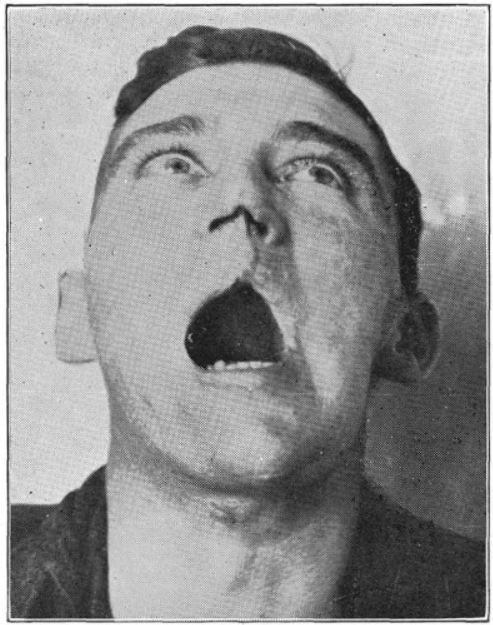

Completely adherent upper lip point of exit of missile.
Figure 33.

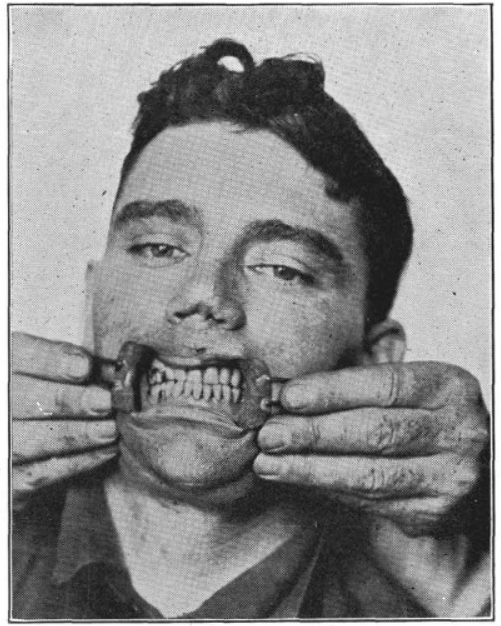

Denture in position.
Figure 32 .

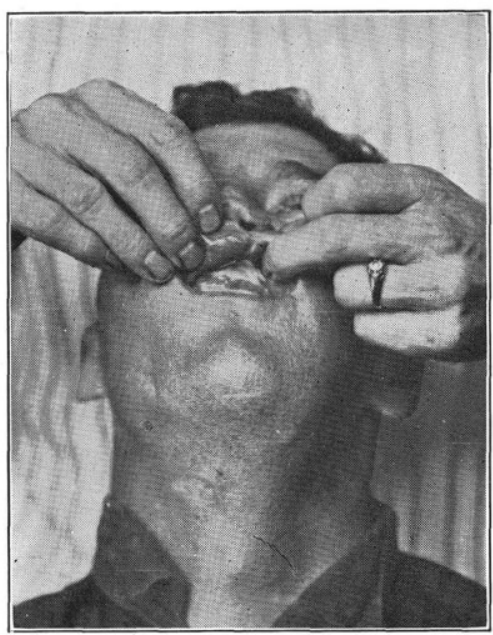

Lip liberated with the skin inlay plainly visible.
Figure 34 .

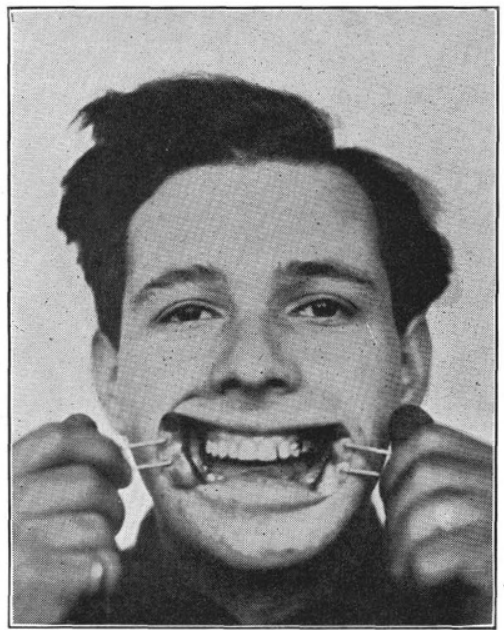

Left lower buccal sulcus obliterated. 
Figure 35.

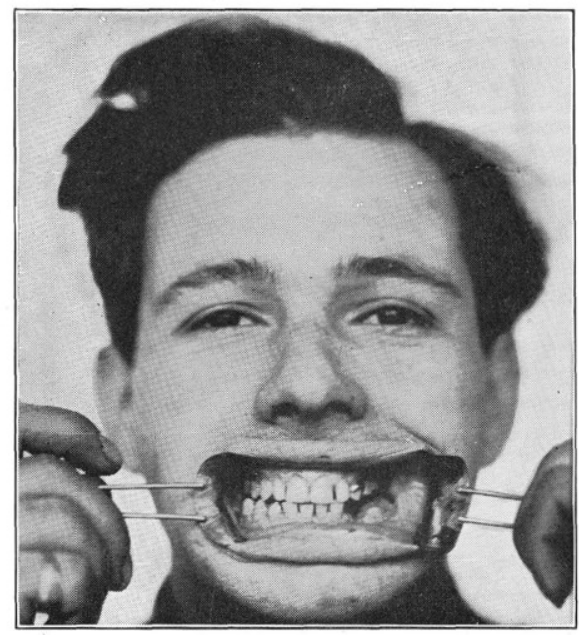

Upper portion of skin inlay visible.

Figure 36.
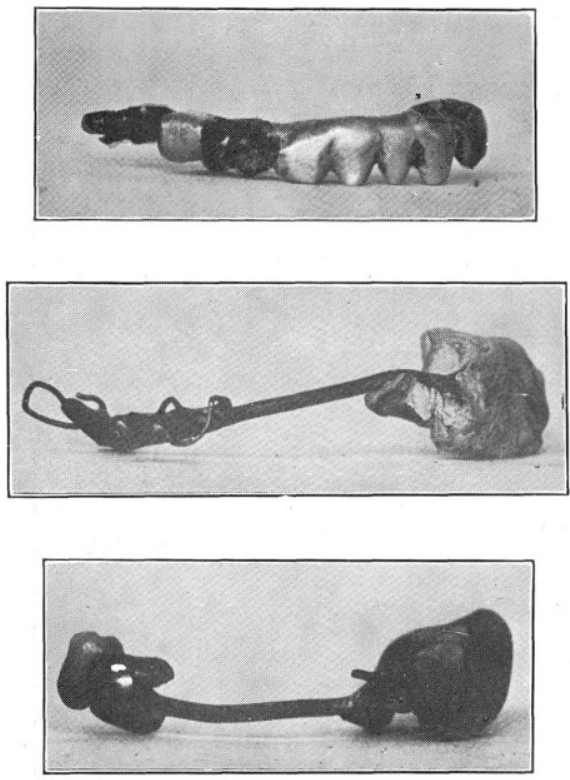

Inferior dental splint used for fracture of mandible, Jackson skin inlay apparatus and denture.
Figure 37.

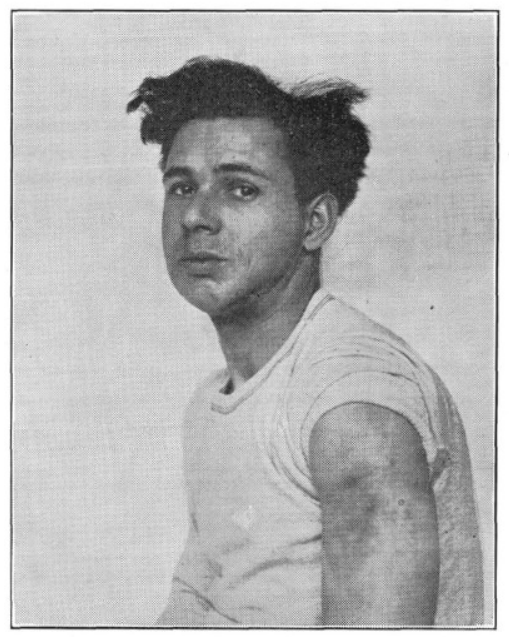

Arm, healed surface from whence Thiersch graft was taken.

Figure 38

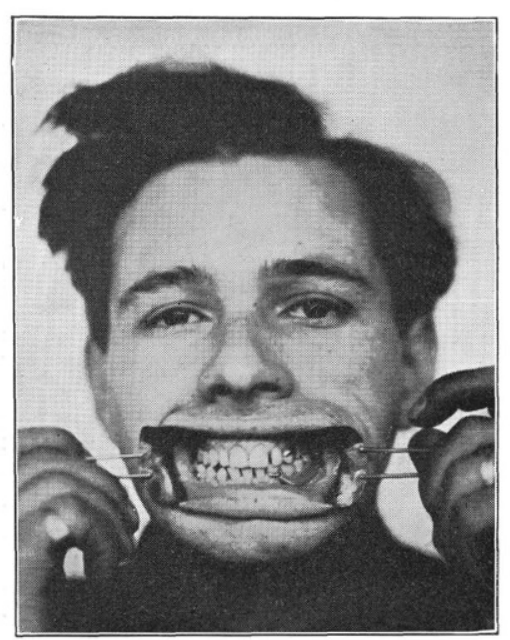

Denture in position. 